\title{
LOS CAMBIOS EN LA POLÍTICA AGROPECUARIA Y LA PROPIEDAD SOCIAL RURAL EN LA FRONTERA NORTE
}

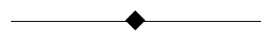

\section{RESUMEN}

El capital privado nacional y extranjero no llegó al campo en los términos en que esperaba el Estado, por lo que los productores del sector social y privado no lograron insertarse en los proyectos especializados que se canalizan a la exportación, generando una descapitalización en los pequeños, medianos y grandes productores que se dedicaban a la producción para el mercado interno. En la frontera norte, la propiedad social sigue siendo importante y seguirá existiendo por mucho tiempo a pesar de los cambios en la política agropecuaria y sobre todo con los cambios al artículo 27 Constitucional de enero de 1992 y a la promulgación de la Ley Agraria en febrero de 1992. La organización de los productores, tanto del sector social como privado, está en crisis y la mayoría de los productores trabajan con sus propios recursos y de manera individual. Esto ha dificultado la integración de proyectos productivos.

El objetivo del presente trabajo es analizar los cambios en la propiedad social rural (ejidos, comunidades, colonias agrícolas y ganaderas) de la frontera norte con la nueva política agropecuaria.

Palabras clave: Estado, política agropecuaria, propiedad social rural, artículo 27 constitucional, productores del sector social.

\section{ABSTRACT}

The national and foreign capital did not arrive to the country in the terms that government was expected; in that way farmers from social and private sector did not get insert into specialized and directed project towards exports. This generated an undercapitalization on small, medium and big farmers that

* Profesor-investigador de el colef, piedras negras, coahuila. Correo electrónico: obezo@comuni-k.com; viofra57@hotmail.com 
work to aim production at domestic market. Communal property on North Border of Mexico is doing important yet, and it will be to exist for long time despite the changes in Agricultural Policy, and basically by the change in Constitutional 27th Article from January 1992, and the passing law about the Agrarian Act on February 1992. The organization of the producers as much as social as private sector is on recession, and most of producers individually work with their own resources or financial means. This has been doing difficult to their integration to productive projects. The aim of this paper is to analyze those under gone changes by the rural property (namely ejidos, communities, agro and farm colonies) of northern Mexico associated to the new agricultural policy.

Keywords: government, agricultural policy, comunal property, constitucional 27th article, farmers from social sector.

\section{INTRODUCCIÓN}

El Estado mexicano decidió cambiar la política agropecuaria a finales de la década delos ochenta y principios dela de los noventa. Con la nueva política, el Estado se retira de las actividades de fomento y apoyo al campo, modifica el marco legal y promuevenuevas figuras asociativas, abriendo espacios a la penetración del capital privado nacional y extranjero, de tal manera que los productores del sector social puedan incorporarseal desarrollo nacional einternacional por medio deproyectos productivos rentablesy competitivos. En otras palabras, con esta política el Estado trata de transferir sus funciones en el campo alos capitales privados nacionales y extranjeros.

México no es un país homogéneo, y está compuesto por regiones agropecuarias con diferentes grados de desarrollo, en el caso dela frontera norte, considerada una región con un alto nivel de desarrollo, dado que cuenta con infraestructura para la producción, para la comercialización, así como recursos naturales (tierra, agua) de muy buena calidad, se pensó que con la nueva política esta región severía favorecida y mejoraría los niveles dedesarrollo, y en particular sebeneficiarían los productores del sector social (ejidos y comunidades) incrementando sus niveles deingreso y condiciones de vida al asociarse con el capital privado nacional o extranjero, además, aumentarían los niveles de producción y productividad al incorporar nuevas tecnologías en los procesos productivos y de comercialización. 
El objetivo del presente trabajo es analizar los cambios en la propiedad social rural (ejidos, comunidades, colonias agrícolas y ganaderas) de la frontera nortecon la nueva política agropecuaria.

En este trabajo, entendemos por propiedad social rural las tierras cedidas por el Estado a los ejidos, comunidades agrarias, colonias agrícolas y ganaderas para su explotación, en común o en forma individual, y que permanecen bajo el control del Registro Agrario Nacional.

En el primer apartado se describen los cambios en la política agropecuaria, en el segund o seanaliza la propiedad social rural en la frontera nortey, final mente, seabordan las conclusi ones y recomendaciones.

\section{LOS CAMBIOS EN LA POLÍTICA AGROPECUARIA}

El modelo económico desustitución de importaciones ponía énfasis en el crecimi ento industrial, lo cual colocaba en un papel subordinado a los demás sectores. En el caso del agro, tenía como prioridad superar los entrabes al progreso dela industria, derivándose deahí las funciones que se le asignaron en el desarrollo económico: proveer bienes-salarios a bajos precios; producir eficientemente materias primas industriales; generar divisas para financiar la importación de bienes de capital para la producción industrial; liberar mano deobra para el mercado de trabajo y contribuir al crecimiento del mercado interno (Gómez, 1996).

Durante varias décadas el Estado seencargó del desarrollo del campo por medio dela inversión y el gasto público. Una gran parte delos programas de desarrollo agrícola, como investigación, asistencia técnica, capacitación, controles sanitarios, créditos y apoyos directos a la producción y comercialización, eran realizados por el Estado. El agro era un sector ampliamentesubsidiado por el Estado. En estecontexto, los productores del sector social (ejidos, comunidades, colonias agrícolas y ganaderas) estaban vinculados y protegidos de manera estrecha por el Estado mediante un marco legal y del aparato institucional defomento y apoyo al campo, que 
se canalizaba antes que otra cosa a las zonas de riego y agroexportadoras.

A partir de 1989, el Estado acelera el proceso dereestructuración económica, y en el caso del sector rural y agrario cambia la política agropecuaria. Esta política consiste fundamentalmente en disminuir de manera drástica los subsidios a los insumos para la producción, desregular los mercados agropecuarios, privatizar las empresas productoras de insumosy, por último, modificar el marco legal agrario. Todo esto obedece principal mentea la reducción dela intervención del Estado en la economía.

En este contexto, el gobierno federal y algunos autores como A rturo Warman y Gustavo Gordillo, que comparten la propuesta, consideraron quecon los cambios en la relación entreel Estado y la sociedad rural, y con la nueva política, sefortalecería el sector rural y agrario y de manera preferente aquel las regiones que contaban con buena calidad de los recursos naturales (tierra-agua-bosques), infraestructura para la producción y comercialización, como es el caso de la frontera norte. En este sentido, es importante abordar brevementelas posiciones del Estado, así como las delos académicos que apoyaban las nuevas políticas para el sector rural y agrario, con el objeto derespaldar el análisisque sehace delos cambios dela política agropecuaria queel Estado realizó a princi pios dela década delos noventa.

Salinas de Gortari (1991), en su iniciativa de reforma al artículo 27 constitucional, propuso tres puntos fundamentales: el primero es dar certidumbre jurídica en el campo, por lo que era necesario terminar con el reparto agrario; el segundo serefiereala capitalización del campo, por lo que eran necesarios cambios en la legislación agraria para atraer y facilitar la inversión privada nacional y extranjera en las proporciones queel campo demanda, para ello se requieretambién nuevas formas de asociación; el tercero y último punto se refierea la protección de la vida ejidal y comunal, reafirmando estas formas de tenencia de la tierra y adecuándolas a las nuevas realidades del país.

Warman (1996) sostuvo quecon la nueva política y las reformas al artículo 27 constitucional se pretende restablecer y recuperar el 
crecimiento y el desarrollo rural; esto es, generar fuentes detrabajo en las actividades agropecuarias y en las comunidades rurales. En esta perspectiva se fincan esperanzas y oportunidades que se habían agotado con la crisis del sector agropecuario y el deterioro de la vida rural. Las cosas en el campo mexicano no podían seguir tal cual, por lo que era urgente su transformación o modernización para lograr su capitalización.

Gordillo (1991) señaló quela nueva política para el campo agrupa y condensa la reforma política, la reforma económica y la reforma social más profunda de las últimas décadas. La no injerencia estatal en la vida de los ejidos y las comunidades permitiría una efectiva apropiación del proceso productivo y esto generaría el incremento en su ingreso y el mejoramiento de las condiciones de vida.

Téllez (1994), sostuvo que con la nueva política para el campo, los nivel es de eficiencia y productividad que exigen las reglas del comercio internacional y delas inversiones privadas, sólo se pueden al canzar en el sector social si se supera el principal reto económico queconsisteen poder integrar parcelas y predios en unidades productivas similares a las grandes extensiones privadas, es decir, setrata deformar áreas compactas. Integrar unidades productivas en las quela extensión delatierra haga atractivo el flujo del capital y detecnología; ésees el papel quesequierequedesempeñelalibre asociación entre ejidatarios, comuneros, pequeños propietarios y empresarios nacionales y extranjeros.

En contraste a la posición del Estado y sus teóricos están las posiciones deal gunos autores con un enfoque crítico. Así, Encinas (1995) sostuvo quea finales de 1991 y sobretodo en 1992, se marcó un cambio en la estrategia gubernamental. Después de sortear los difíciles momentos iniciales y reafirmada la estabilidad económica, las medidas delegitimación y concertación social adoptadas en los primeros tres años dela administración sal inista dejaron deser prioritariasy perdieron influencia en las definiciones políticas, ocupando esta posición los grandes grupos empresariales.

A ppendini (1995) planteó que esta política no niega la participación social del campo, sin embargo, los productores ejidales son 
tratados de la misma manera que el resto de los empresarios, es decir, no tienen un tratamiento preferencial, el cual reducelos espacios de participación de los ejidos y las comunidades en el mercado. A nteesta situación, la política tiende a fortalecerseen el sector privado como agentedela transformación del agro, subordinando a los ejidos y las comunidades en la organización productiva.

Zermeño (1992) señaló que esta política que planteaba el Estado para el campo es producto de la negociación y firma de un Tratado deLibreComercio con Estados Unidosy Canadá; en estecontexto, por más exitosa quefuera la política agroexportadora derivada del TLC, dejaría excluidos a la mayoría delos productores, ya quecuando mucho $20 \%$ de la superficie cultivada del país se destinaría a productos deexportación. En el restante $80 \%$ dela superficiecultivada se ubicarían entre 85 y $90 \%$ de los productores del sector social y privado del agro, los cuales serían presionados a diversificar sus actividades; sin embargo, el sector urbano-industrial no ofreceal ternativas en el corto y mediano plazos.

Fritscher (1993) afirmó queen los primeros tres años dela administración salinista seconfiguró cabalmenteel proyecto neoliberal para el campo mexicano; esto lo demuestra al ensanchar la apertura comercial unilateral, la privatización de las empresas paraestatal es queapoyaban al sector agropecuario, el retiro estatal en las tareas de promoción y fomento, la eliminación delos precios de garantía y final mente la aprobación de una legislación agraria queabrelos espacios ejidales a terceros. En consecuencia, el Estado se retira de manera acelerada del campo y busca que éste se convierta en un espacio de la inversión privada; sin embargo, para la autora el retiro del Estado del campo y la entrada de capital es privados no soluciona el problema del sector agropecuario en crisis desde hacemucho tiempo, mismo que seha agudizado en los últimos años por las políticas macroeconómicas aplicadas por el gobierno sal inista. Esto hacequeel campo mexicano no ofrezca, salvo algunas excepciones, condiciones de rentabilidad a los capitales privados nacionales y extranjeros.

El agro no puede ser tratado con los mismos instrumentos que los demás sectores de la economía. Su dependencia de recursos 
natural es, por lo general no mejorables y en muchos casos sujetos a elementos al eatorios, como el clima, lo convierten en un sector que ofrece pocas garantías a la inversión capitalista nacional o extranjera; de ahí quesu desempeño ha debido estar históricamentevinculado y protegido por el Estado. La posición deéstedeapoyar un solo proyecto para el agro con base en la especial ización productiva y las ventajas comparativas, dejaría excluido al grueso de los productores del sector social de la agricultura e incorporaría al sector delos excluidos a pequeños, medianos y grandes productoresprivados.

Cartón deGrammont (1996) sostuvo que la nueva política dividió al campo en dos sectores: un sector empresarial, moderno y competitivo en el mercado internacional, y otro sector compuesto por productores atrasados y de subsistencia apoyados por limitados programas de combate a la pobreza. En el sector empresarial moderno y competitivo, el Estado busca crear un nuevo actor productivo capaz de sustituir las funciones gubernamentales para invertir en el campo. Con esta política, el Estado no solamenteexcluye a los productores del sector social del campo, sino también al agricultor, el hombre emprendedor del campo, el productor familiar quefueagentepreferencial del desarrollo agrícola; ahora el Estado espera ver la aparición degrandes empresas directamentevinculadas con la inversión agroindustrial, que resuelvan el problema de rezago y crisis del campo.

Independientemente de las distintas posiciones en relación con los cambios dela política para el campo, es importante mencionar queen el país existen regiones agropecuarias con diferentes grados de desarrollo, y al interior de las regiones se encuentran ejidos y comunidades que cuentan con buena infraestructura y recursos naturales debuena calidad (tierra-agua-bosques); ejidosy comunidades con poca infraestructura y con recursos naturales demediana calidad; ejidos y comunidades con recursos naturales de mala calidad, sin infraestructura y sin agua, es decir, 100\% detemporal . En este contexto, aparentemente a los capital es privados nacionales y extranjeros les interesa invertir en aquel los ejidos y comunidades quetienen buena infraestructura y recursos naturales debuena 
calidad; en cambio, aquellos ejidos y comunidades con condiciones medias de desarrollo que requieren fuertes inversiones para rehabilitar la infraestructura, así como los eji dos marginados que son la mayoría del país, no son deinterés de los capital es privados nacionales y extranjeros.

A parentemente, la nueva política pod ría tener un impacto positivo en los productores del sector social dela frontera norte, considerada como una región con un al to nivel de desarrollo.

\section{LA PROPIEDAD SOCIAL RURAL EN LA FRONTERA NORTE}

La región frontera norteincluyelos estados deBaja California, Sonora, Chihuahua, Coahuila, Nuevo León y Tamaulipas.

Al abordar el tema del sector rural y agrario de la frontera norte, inmediatamentenos imaginamos un sector moderno, desarrollado y altamente tecnificado; sin embargo, la real idad es otra, ya queesta región no es homogénea tanto en la disponibilidad de recursos naturales como en la estructura agraria. En la región existe una estructura agraria compuesta por propietarios privados, ejidatarios, comuneros, y colonos agrícolas y ganaderos, a pesar de los cambios en el marco legal que pretendían transformar esta estructura agraria.

Hasta antes de los cambios en la pol ítica agropecuaria y agraria, la organización de la administración pública agropecuaria en la frontera nortetenía una cobertura casi total, lo que permitió favorecer el desarrollo de esta estructura productiva al apoyar con créditos, asistencia técnica, abastecimiento de insumos, investigación, seguro agropecuario, organización, capacitación, comercialización, creación deinfraestructura hidroagrícola y precios degarantía para al gunos productos. Esta estructura productiva adqui rió gran relevancia hasta 1989, produciendo granos básicos, granos industriales, frutas, hortalizas, carne, leche, huevo, tanto para el mercado interno como para el externo. Esta estructura generó empleos eingresos para la mayor parte dela población rural.

La nueva política para el campo redujo la participación del Estado en la producción y comercialización de insumos, dejando los 
espacios al sector privado y al sector social por medio desus organizaciones.

El Estado, a través de las instituciones del sector agropecuario, promovió la asociación en participación entre los productores del sector social y los capital es privados nacionales o extranjeros. Los resultados deesta promoción en la frontera norteno fueron los que esperaba el Estado, ya que los capitales que se interesaron en el campo fueron aquel los que estaban vinculados a las actividades agropecuariasy agroindustriales; los demás capital es no se interesaron, ya que las tasas deganancia resultan más atractivas en otras ramas de la economía. Esto redujo las expectativas del Estado al apostar a la capitalización del campo mediante la penetración de capitales privados nacionales y extranjeros. Como ejemplo tenemos el Proyecto Vaquerías que promovió el Estado a través de la Secretaría deA gricultura y Recursos Hidráulicos entrela empresa agroindustrial Galletera Mexicana S. A. (Gamesa) y los ejidos de los municipios de Linares y Montemorelos en el estado de Nuevo León. Otro ejemplo es el proyecto de asociación entre la empresa Bimbo y los ejidos productores detrigo en el nortede Coahuila.

Los cambios en la política agropecuaria estaban encaminados a aquellas regiones del país con niveles al tos de desarrollo como es el caso de la frontera norte, debido a la disponibilidad debuena infraestructura hidroagrícola para la producción y la comercialización, recursos naturales debuena calidad (tierra-agua-bosques), lo cual motivaría a los capitales privados nacionales y extranjeros para invertir en la región; sin embargo, dada la heterogeneidad deésta, existen zonas con las características mencionadas, otras con condiciones medias de desarrollo, así como áreas sin infraestructura para el desarrollo delas actividades agropecuarias, tales como las zonas ixtleras y candelilleras de los estados de Chihuahua, Coahuila, Nuevo León y Tamaulipas.

Bajo estas condiciones de la frontera norte, los capitales privados nacional es y extranjeros queseinteresaron en el campo fueron los capitales agropecuarios y agroindustriales que de al guna manera ya estaban presentes en las zonas con al to grado dedesarrollo (como el sur de Sonora, Baja California, y la zona de N avidad, en 
Nuevo León), y lo único quehicieron fue ampliar su presencia por medio de la renta de tierras del sector social o anexando a sus empresas tierras de propiedad privada; sin embargo, la mayoría de los productores del sector social y privado que producían para el mercado interno quedaron excluidos de estenuevo proyecto para el campo.

En virtud dequesolamenteun pequeño número deproductores agrícolas pudo asociarse con los capitales privados nacionales y extranjeros, dejando al margen los esfuerzos deejidos y comunidades, así como de pequeños, medianos eincluso grandes productores privados, el Estado se vio obligado a promover en la región políticas públicas Ilamadas compen satorias para que de al guna forma seapoyara a los productores rurales del sector social y privado, tales como el Programa deA poyo al Campo, Alianza para el Campo, Programas de Empleo Temporal , A poyos a la Comercial ización Agropecuaria, así como Programas de Organización y Capacitación para los productores rurales del sector social por medio delas instituciones del sector agropecuario, con el fin de integrarlas al desarrollo productivo regional.

Según la Secretaría de la Reforma Agraria (2002), la capacitación servirá para quelos sujetos agrarios conozcan sus derechos y obligaciones, además de que podrán adquirir los conocimientos necesarios para utilizar la tecnología productiva, el manejo de la información de mercado para mejorar su capacidad de compra de insumos y venta de productos sin la intervención de los interme diariosy con ello poder capitalizarse, tener capacidad deahorro y aprovechar las ventajas comparativas con quecuentan los núcleos agrarios. Asimismo, la organización delos sujetos agrarios les permitirá que se unan entre ellos o con terceros para que mediante figuras asociativas las unidades de producción sean mayores y aprovechen las economías de escala, generen proyectos productivos y obtengan una mejora en su poder de negociación y comercialización.

El resultado dela organización y capacitación en la frontera norte se muestra en el cuadro 1. Este cuadro muestra un total de 2625 figuras asociativas, las cuales se dividen de la siguiente manera: 
1063 Sociedades de Solidaridad Social; 1308 Sociedades deProducción Rural; 15 Uniones de Sociedades de Producción Rural; 180 Uniones deEjidos y Comunidades; 35A sociaciones Rurales delnterés Colectivo; seis Federaciones de Sociedades deSolidaridad Social, y 18 Sociedades Mercantiles.

En el cuadro 1 se observa que los estados que destacan en la formación defiguras asociativas son Tamaulipas y Sonora, mientras que el que muestra menos organización para la producción y comercialización es Nuevo León. Los tipos de organización que con mayor frecuencia se presentan en orden deimportancia son las Sociedades de Producción Rural, Ias Sociedades de Solidaridad Social y las Uniones de Ejidos y Comunidades, las cuales en su mayoría seencuentran en Tamaulipas y Sonora; por lo queserefiere a las A sociaciones Rurales de Interés Colectivo, destacan los

CUADRO 1. Figuras organizativas para la producción y comercial ización en la frontera norte.

\begin{tabular}{|lrrrrrrrr|}
\hline Entidad federativa & SSS & SPR & USPR & UEYC & ARIC & FSSS & SM & Total \\
\hline Baja California & 9 & 94 & 1 & 15 & 5 & 0 & 2 & 126 \\
Chihuahua & 39 & 37 & 2 & 22 & 5 & 0 & 1 & 106 \\
Coahuila & 231 & 21 & 0 & 29 & 1 & 0 & 2 & 284 \\
NuevoLeón & 31 & 9 & 0 & 12 & 2 & 0 & 5 & 59 \\
Sonora & 321 & 1046 & 10 & 57 & 18 & 6 & 7 & 1465 \\
Tamaulipas & 432 & 101 & 2 & 45 & 4 & 0 & 1 & 585 \\
\hline Total & 1063 & 1308 & 15 & 180 & 35 & 6 & 18 & 2625 \\
\hline
\end{tabular}

Fuente: Elaboración propia con datos del Registro Agrario Nacional, junio de 2000.

SSS: Sociedad de Solidaridad Social

SPR: Sociedad de Producción Rural

USPR: Unión de Sociedades de Producción Rural

UEYC: Unión de Ejidos y Comunidades

ARIC: A sociación Rural de Interés Colectivo

FSSS: Federación de Sociedades de Solidaridad Social

SM: Sociedad Mercantil

http:/ / www.sra.gob.mx/ monag/ bcweb/ s7Bajacalifornia.htm

http:/ / www.sra.gob.mx/ monag/ coahweb/ s7Coahuila.htm http:/ / www.sra.gob.mx/ monag/ chiweb/ s7Chihuahua.htm http:/ / www.sra.gob.mx/ monag/ nlweb/ s7NuevoLeon.htm http:/ / www.sra.gob.mx/ monag/ tamweb/ sTTamaulipas.htm http:/ / www.sra.gob.mx/ monag/ sonweb/ s7Sonora.htm 
estados deSonora, Baja California y Chihuahua; por último, donde más Sociedades Mercantiles sehan formado es en Sonora y Nuevo León.

La visión del Estado respecto a que la organización y capacitación, así como las políticas públi cas compensatorias sean los factores que impulsen el desarrollo del sector agropecuario y de los productores excluidos del nuevo proyecto, hasta ahora, desafortunadamente, no han dado los resultados esperados, lo queserefleja en la profunda crisis en que seencuentran inmersos los productores rurales dela frontera norte.

En lo querespecta a la situación queestán viviendo los productores de la frontera norte, al gunos líderes campesinos como el señor N oéTapia, presidentedel ComitéRegional Campesino del Municipio deMatamoros, Tamaulipas, el señor JoséLuis Razo, Presidente del ComitéRegional Campesino del Municipio deCaborca, Sonora, el señor Leonel Falcón Garza, Presidente del Comité Regional Campesino del Municipio de Reynosa, Tamaulipas, sostienen que antes de la reforma al artículo 27 los productores del sector social seencontraban mejor organizados, tenían más y mejores apoyos y vendían sus productos a un buen precio; sin embargo, con las reformas al campo sequedaron sin subsidios, sin apoyos y ahora trabajan demanera individual y con sus propios recursos, mientras que la mayor partedesu producción es para el autoconsumo y la poca quelogran vender es a precio muy bajo.

Por otra parte, otros productores han sido pulverizados por el mercado externo, los precios de sus productos están muy bajos, debido a la fal ta de protección, ya queel gobierno federal permite altas importaciones en épocas decosecha, lo que trae como consecuencia una caída de precios y esto ha generado un proceso de descapitalización acelerado de los productores.

En otras zonas de la frontera norte la falta de apoyos y la no rentabilidad de las actividades agropecuarias han hecho que la organización para la producción esté desapareciendo. El caso concreto es el de las Uniones de Ejidos, de las cuales la mayoría han desaparecido y las pocas que todavía funcionan operan entre 10 y $20 \%$ de su capacidad. Otras organizaciones, como las Sociedades 
de Solidaridad Social, se constituyeron formalmente, pero en la realidad no están funcionando (señor Noé Tapia, presidente del Comité Regional Campesino del Municipio de Matamoros, Tamaulipas, marzo de2002).

Así como las organizaciones de primer nivel se encuentran con serios problemas de funcionamiento, también las organizaciones desegundo y tercer nivel (A sociaciones Rurales del nterés Colectivo) enfrentan serios obstáculos en la operación delas diversas actividades agropecuarias. La mayoría de los campesinos trabajan de manera individual con sus propios recursos, lo que genera que se incrementen los costos y se reduzcan o desaparezcan las ganancias, ya que compran sus insumos al último intermediario de la cadena y venden sus productos al primer intermediario. La descapitalización de los productores ha sido la causa principal del fracaso de este tipo de organizaciones. Es importante resaltar la opinión deal gunos líderes campesinos dela frontera norte: "con la reforma al artículo 27 y con la nueva ley la gente pensó que ya somos autosuficientes, y entonces lejos de buscar nuevos mecanismos para estar más unificados y obtener mayores rendi mientos o mayor beneficio en la comercialización, no los hemos tenido. Nos hemos distanciado más unos de otros." "A horita como estamos trabajando con el Tratado de Libre Comercio, si nuestros costos de producción son de 2 mil pesos por hectárea, es lo mismo quesacamos dela venta denuestro producto, no hay ganancias, el mercado externo nos ha pegado muy duro, ha bajado mucho el precio de nuestro producto." “En la región deM atamoros, la Unión deEjidos está trabajando en un 10 por ciento de su capacidad, no tenemos capacidad económica para unirnos en ese sentido, ya que desiete años para acá no hay utilidades, y con lo poco que sacamos estamos sobreviviendo." "N osotros en Caborca tenemos la Unión de Ejidos Luis Echeverría. Lo que pasa es quela Unión está deteriorada, la Unión se conformaba de 33 ejidos, ahorita quedamos 15y de los 15 estamos sectorizados en grupitos, en un ejido antes eran grupos de 50 personas, y ahora son grupos de cinco o diez personas." "En A gua Prieta ya no existe la Unión de Ejidos. Esta Unión se dedicaba a comprar insumos y distribuirlos con crédito de 
Banrural, actividades que ya no desempeña" (señor N oé Tapia, presidentedel ComitéRegional Campesino del Municipio deMatamoros, Tamaulipas; señor José Luis Razo, presidentedel Comité Regional Campesino del Municipio de Caborca, Sonora; señor Gildardo Duarte, Presidente del Comité Regional Campesino del Municipio de A gua Prieta, Sonora, marzo-abril de 2002).

Todo lo anterior ha dificultado la integración de proyectos productivos en los ejidos y las comunidades, y ha obligado a los productores del sector social a diversificar sus actividades, ocupándose en la industria maquiladora, en actividades propias de las ciudades cercanas a sus comunidades o a migrar a Estados Unidos y los productores de edad avanzada se dedican al trabajo delatierra.

Las razones defondo que perseguía el Estado con el cambio en la política agropecuaria y agraria eran que la propiedad social se transformara en propiedad privada y la mayoría de la población rural migrara hacialas ciudades, dejando sólo de un tres a un cinco por ciento en el campo; sin embargo, después de más de 10 años se observa quela propiedad social y la población rural siguen manteniendo un peso importante en la frontera norte.

En estesentido, deacuerdo con el Instituto N acional deEstadística, Geografía el nformática (INEGI), la población rural en la frontera norte es ligeramente superior a los 2 millones de habitantes y representa $13.13 \%$ de la población total. Las entidades con mayor población rural son: Chihuahua con $17.45 \%$, Sonora con $16.91 \%$ y Tamaulipas con $14.57 \%$ (véase cuadro 2).

Deacuerdo con datos deINEGI y la Procuraduría A graria, aproximadamente $35 \%$ de la población rural se ocupa en actividades agropecuarias y en su mayoría son personas mayores de 40 años; en cambio, el resto dela población en edad detrabajar y menores de 40 años se ocupan en la industria maquiladora, otros trabajan en las ciudades cercanas a su comunidad y como último recurso migran hacia Estados Unidos; hay entidades donde la migración rural hacia la Unión A mericana tieneun peso importante, como es el caso del norte deNuevo León. 
CUADRo 2. Participación de la población rural en la frontera norte.

\begin{tabular}{|lrrrrr|}
\hline $\begin{array}{l}\text { Entidad } \\
\text { federativa }\end{array}$ & $\begin{array}{r}\text { Población } \\
\text { total }\end{array}$ & $\begin{array}{r}\text { Población } \\
\text { urbana }\end{array}$ & $\%$ & $\begin{array}{r}\text { Población } \\
\text { rural }\end{array}$ & $\%$ \\
\hline Baja California & 2487700 & 2281068 & 91.69 & 20663 & 28.31 \\
Chihuahua & 3047867 & 2515961 & 82.55 & 531906 & 17.45 \\
Coahuila & 2295808 & 2052825 & 89.42 & 242983 & 10.58 \\
NuevoLeón & 3826240 & 3573966 & 93.41 & 252274 & 6.59 \\
Sonora & 2213370 & 1839135 & 83.09 & 374235 & 16.91 \\
Tamaulipas & 2747114 & 2346734 & 85.43 & 400380 & 14.57 \\
\hline Total & 16816099 & 14609689 & 86.87 & 2008410 & 13.13 \\
\hline
\end{tabular}

FunNte: Elaboración propia con datos el INEGI. XII Censo General de Población y Vivienda, 2000. Resultados preliminares.

Los ejidatarios y sus familias comprenden la mayor parte de la población rural dela frontera norte. Losingresos por ejidatario son muy heterogéneos, sin embargo, en promedio, 50\% delosejidatarios reciben más deun salario mínimo. En el cuadro 3sepuedeobservar que las entidades que destacan con mayores ingresos son Baja California y Sonora, esto se debe seguramenteal grado demodernización y al dinamismo de las actividades agropecuarias.

CUADRo 3. Ingreso de los ejidatarios por entidad federativa en la frontera norte.

\begin{tabular}{|lrrrrr|}
\hline $\begin{array}{l}\text { Entidad } \\
\text { federativa }\end{array}$ & $\begin{array}{r}\text { Ingreso de } \\
\text { 1SM (\%) }\end{array}$ & $\begin{array}{r}\text { Ingreso de más } \\
\text { de 1 hasta 5 } \\
\text { SM (\%) }\end{array}$ & $\begin{array}{r}\text { Ingreso de más } \\
\text { de5SM (\%) }\end{array}$ & $\begin{array}{r}\text { No recibe } \\
\text { ingreso (\%) }\end{array}$ & $\begin{array}{r}\text { Número de } \\
\text { ejidatarios }\end{array}$ \\
\hline Baja California & 15.70 & 68.50 & 12.90 & 2.90 & 20628 \\
Chihuahua & 22.50 & 43.50 & 4.80 & 29.00 & 84456 \\
Coahuila & 41.40 & 41.40 & 2.40 & 14.60 & 71354 \\
NuevoLeón & 29.70 & 40.50 & 5.40 & 24.30 & 39990 \\
Sonora & 17.10 & 70.70 & 7.30 & 4.90 & 74066 \\
Tamaulipas & 35.00 & 38.00 & 11.00 & 16.00 & 81211 \\
\hline Total & 27.06 & 50.35 & 7.30 & 13.61 & 679410 \\
\hline
\end{tabular}

FuENTE: Elaboración propia con datos de la Procuraduría Agraria, marzo de 2000.

$\mathrm{SM}=$ Salario Mínimo.

http:/ / www.sra.gob.mx/ monag/ bcweb/ s3abcn.htm

http:/ / www.sra.gob.mx/ monag/ coahweb/ s3acoa.htm

http:/ / www.sra.gob.mx/ monag/ chiweb/ s3achi.htm

http:/ / www.sra.gob.mx/ monag/ nlweb/ s3anl.htm

http:// www.sra.gob.mx/ monag/ tamweb/ s3atam.htm

http:/ / www.sra.gob.mx/ monag/ sonweb/ s3ason.htm 
Esimportantemencionar quelos dosúltimos rangos queutiliza la Procuraduría Agraria para clasificar los ingresos de los ejidatarios esconden la realidad, pero, aun así, se detectó queBaja California y Sonora destacan con los ingresos más altos; en cambio, en el resto de los estados predominan los ingresos entre cero y un salario mínimo. Adicional a los ingresos de los ejidatarios, una buena parte de las familias recibeapoyos económi cos delos hijos quetrabajan en la industria maquiladora o en al gunas otras actividades desempeñadas en el medio urbano, así como del trabajo temporal en Estados Unidos.

La edad promedio de los ejidatarios dela frontera nortees de 50 años (véase cuadro 4), lo que demuestra que la mayoría no tiene otra opción y debe dedicarse al trabajo de su parcela, además de que algunos ejidatarios están incorporados al programa oficial Procampo.

En lo referentea la estructura agraria, los cambios que se hicieron al artículo 27 constitucional en 1992 y la promulgación de la Ley Agraria en esemismo año, no tuvieron un impacto significativo en el cambio dela estructura agraria de la frontera norte.

Cuadro 4. Edad promedio de los ejidatarios en la frontera norte.

\begin{tabular}{|lcc|}
\hline Entidad federativa & $\begin{array}{c}\text { M asculino } \\
\text { (años) }\end{array}$ & $\begin{array}{c}\text { Femenino } \\
\text { (años) }\end{array}$ \\
\hline Baja California & 51.7 & 55.2 \\
Chihuahua & 49.3 & 53.9 \\
Coahuila & 51.7 & 58.3 \\
Nuevo León & 50.9 & 58.7 \\
Sonora & 52.5 & 55.3 \\
Tamaulipas & 51.8 & 54.9 \\
\hline
\end{tabular}

Fuente: Elaboración propia con datos de la Procuraduría Agraria. "Tipología sujetos agrarios PROCEDE", RAN-PA. http:/ / www.sra.gob.mx/ monag/ bcweb/ s3Bajacalifornia.htm

http:/ / www.sra.gob.mx/ monag/ coahweb/ s3Coahuilal.htm

http:/ / www.sra.gob.mx/ monag/ chiweb/ s3Chihuahua.htm http:/ / www.sra.gob.mx/ monag/ nlweb/ s3NuevoLeon.htm http:/ / www.sra.gob.mx/ monag/ tamweb/ s3Tamaulipas1.htm http:/ / www.sra.gob.mx/ monag/ sonweb/ s3Sonoral.htm 
En estesentido, en la región sigue existiendo un equilibrio entre la superficiequeocupa la propiedad social (ejidos, comunidadesy colonias agrícolas y ganaderas) y la propiedad privada. El cuadro 5 muestra que la propiedad privada ocupa $52 \%$ de la superficie total dela frontera nortey el $48 \%$ restantecorrespondea la propiedad social . A excepción de Baja Cal ifornia, en la que predomina la propiedad social (95.17\%), en la mayoría de las entidades existe más o menos un cierto equilibrio entre la propiedad social y la propiedad privada, lo que demuestra que la propiedad social seguirá existiendo por mucho tiempo en esta región, a pesar delos esfuerzos del Estado por privatizarla. La propiedad social seguirá existiendo en diversas modalidades: en una primera modal idad se encuentran los ejidos y las comunidades agrarias queaún trabajan latierra directamentey dependen deella como una fuentedeingresos importante; en una segunda modalidad seencuentran los ejidos y las comunidades agrarias que por condiciones de ubicación geográfica y sistemas de producción para la exportación, prefieren rentar sus tierras al capital privado nacional o extranjero dados los escasos apoyos que otorga el Estado; sin embargo, la propiedad

CUADRo 5. Participación dela propiedad social y privada en la superficie de la frontera norte.

\begin{tabular}{|c|c|c|c|c|c|}
\hline $\begin{array}{l}\text { Entidades } \\
\text { federativas }\end{array}$ & $\begin{array}{l}\text { Propiedad } \\
\text { total (has) }\end{array}$ & $\begin{array}{l}\text { Propiedad } \\
\text { social (has) }\end{array}$ & $\%$ & $\begin{array}{r}\text { Propiedad } \\
\text { privada (has) }\end{array}$ & $\%$ \\
\hline Baja Californi & a 6309030 & 6004481 & 95.17 & 304549 & 4.83 \\
\hline Coahuila & 14852343 & 6868370 & 46.24 & 7983973 & 53.76 \\
\hline Chihuahua & 22845478 & 10636105 & 46.55 & 12209373 & 53.45 \\
\hline Nuevo León & 6315445 & 2163948 & 34.26 & 4151497 & 65.74 \\
\hline Sonora & 15820742 & 6739112 & 42.60 & 9081630 & 57.40 \\
\hline Tamaulipas & 7362078 & 2891560 & 39.28 & 4470518 & 60.72 \\
\hline Total & 73505116 & 35303576 & 48.02 & 38201540 & 51.98 \\
\hline
\end{tabular}

Fuente: Elaboración propia con datos del Registro Agrario NacionalSubsecretaría de Ordenamiento de la Propiedad Rural.

http:/ / www.sra.gob.mx/ monag/ bcweb/ s2Bajacalifornia.htm http:/ / www.sra.gob.mx/ monag/ coahweb/ s2Coahuilal.htm http:/ / www.sra.gob.mx/ monag/ chiweb/ s2Chihuahua.htm http:/ / www.sra.gob.mx/ monag/ nlweb/ s2Nuevo\%20Leon1.htm http:/ / www.sra.gob.mx/ monag/ tamweb/ s2\%20Tamaulipas.htm http:/ / www.sra.gob.mx/ monag/ sonweb/ s2Sonora2.htm 
sigue perteneciendo al régimen depropiedad social, es decir, están dentro del Registro A grario Nacional. Esta propiedad social rentada al capital privado seguirá siendo propiedad social, mientras no cambie su registro, es decir, al Registro Público dela Propiedad.

Al analizar de manera particular la propiedad social, sedetectó que predomina la propiedad ejidal, el cuadro 6 muestra la distribución dela propiedad social y en él seobserva quelosejidos detentan $87.90 \%$ dela superficietotal dela propiedad social, mientras quela propiedad comunal y las colonias agrícolas y ganaderas poseen 6.40 y $5.70 \%$ respectivamente.

En relación con los ejidos, en la frontera norte existen 4857, con una superficie de 31026490 hectáreas y una superficie promedio por ejido de 6388 hectáreas. Demanera desagregada por cada una delas entidadesfederativas quecomponen la región, en el cuadro 7 se puede observar que Baja California es la entidad que tiene el menor número deejidosy Tamaulipasla mayor.

Sin embargo, en términos desuperficiela situación cambia, ocupand o el primer lugar Chihuahua con 30.6\% dela superficieejidal, seguido por Coahuila, Baja California y Sonora con 20.8, 17.8 y $16.9 \%$ respectivamente. En términos de superficie promedio por ejido, el primer lugar lo ocupa Baja California con 24982 hectáreas, seguido por Chihuahua y Coahuila con 10466 y 7298 hectáreas respectivamente (véase cuadro 7). Por último, en cuanto a la calidad de recursos naturales como tierra y agua, sobresalen Baja Cal ifornia, Sonora y Chihuahua.

Respecto a las comunidades, en esta región existen 145 con una superficie de 2261323 hectáreas y una superficie promedio por comunidad de 15595 hectáreas. Demanera segregada en cada una delas entidadesfederativas, en el cuadro 8seobserva queSonora y Chi huahua son los estad os que en conjunto absorben más de $80 \%$ tanto en número de comunidades como en superficie; también es importante destacar que las comunidades de mayor peso son los indígenas yaquis y mayos en Sonora y los tarahumaras en Chi huahua. A simismo, en la superficie promedio por comunidad destacan los estados de Baja Cal ifornia, Tamaulipas y Sonora; sin 
CUADRO 6. Distribución de la propiedad social en la frontera norte.

\begin{tabular}{|lrrrrrrrr|}
\hline $\begin{array}{l}\text { Entidades } \\
\text { federativas }\end{array}$ & $\begin{array}{r}\text { Propiedad Propiedad } \\
\text { social } \\
\text { (has) }\end{array}$ & $\begin{array}{r}\text { ejidal } \\
\text { (has) }\end{array}$ & $\begin{array}{r}\text { \% } \\
\text { Propiedad } \\
\text { comunal } \\
\text { (has) }\end{array}$ & $\begin{array}{r}\text { \% } \\
\text { Propiedad } \\
\text { de colonias } \\
\text { (has) }\end{array}$ & $\%$ \\
\hline Baja California & 6004481 & 5546176 & 92.36 & 221619 & 3.69 & 236686 & 3.94 \\
Coahuila & 6868370 & 6422382 & 93.50 & 11504 & 0.16 & 434484 & 6.32 \\
Chihuahua & 10636105 & 9472433 & 89.05 & 590800 & 5.55 & 572872 & 5.38 \\
Nuevo León & 2163948 & 1922037 & 88.82 & 123996 & 5.73 & 117915 & 5.44 \\
Sonora & 6739112 & 5258972 & 78.03 & 1133639 & 16.82 & 346501 & 5.14 \\
Tamaulipas & 2891560 & 2404490 & 83.15 & 179765 & 6.21 & 307305 & 10.62 \\
\hline Total & 3530357631026490 & 87.90 & 2261323 & 6.40 & 2015763 & 5.70 \\
\hline
\end{tabular}

Fuente: Elaboración propia con datos del Registro Agrario NacionalSubsecretaría de Ordenamiento de la Propiedad Rural.

http:/ / www.sra.gob.mx/ monag/ bcweb/ s2Bajacalifornia.htm http:/ / www.sra.gob.mx/ monag/ coahweb/ s2Coahuilal.htm http:/ / www.sra.gob.mx/ monag/ chiweb/ s2Chihuahua.htm http:/ / www.sra.gob.mx/ monag/ nlweb/ s2Nuevo\%20Leon1.htm http:/ / www.sra.gob.mx/ monag/ tamweb/ s2\%20Tamaulipas.htm http:/ / www.sra.gob.mx/ monag/ sonweb/ s2Sonora2.htm 
CUADRo 7. Número de ejidos en la frontera nortey participación por entidad federativa en la superficie ejidal.

\begin{tabular}{|lrrrrrr|}
\hline $\begin{array}{l}\text { Entidad } \\
\text { federativa }\end{array}$ & Ejidos & $\%$ & $\begin{array}{r}\text { Superficie } \\
\text { (has) }\end{array}$ & $\%$ & $\begin{array}{r}\text { Superficie } \\
\text { promedio } \\
\text { (has) }\end{array}$ \\
\hline Baja California & 222 & 4.61 & 5546176 & 17.8 & 24982.80 \\
Coahuila & 880 & 18.11 & 6422382 & 20.8 & 7298.16 \\
Chihuahua & 905 & 18.62 & 9472433 & 30.6 & 10466.77 \\
Nuevo León & 593 & 12.20 & 1922037 & 6.19 & 3241.20 \\
Sonora & 934 & 19.22 & 5258972 & 16.9 & 5630.60 \\
Tamaulipas & 1323 & 27.22 & 2404490 & 7.8 & 1817.45 \\
\hline Total & 4857 & & 31026490 & 100.0 & 6388.00 \\
\hline
\end{tabular}

Fuente: Elaboración propia con datos del Registro Agrario NacionalSubsecretaría de Ordenamiento de la Propiedad Rural septiembre de 2000.

http:/ / www.sra.gob.mx/ monag/ bcweb/ s2Bajacalifornia.htm http:/ / www.sra.gob.mx/ monag/ coahweb/ s2Coahuilal.htm http:/ / www.sra.gob.mx/ monag/ chiweb/ s2Chihuahua.htm http:/ / www.sra.gob.mx/ monag/ nlweb/ s2N uevo\%20Leon1.htm http:/ / www.sra.gob.mx/ monag/ tamweb/ s2\%20Tamaulipas.htm http:/ / www.sra.gob.mx/ monag/ sonweb/ s2Sonora2.htm

CUADRO 8. Número de comunidades en la frontera norte y participación por entidad federativa de la superficie comunal.

\begin{tabular}{|lrrrrr|}
\hline $\begin{array}{l}\text { Entidad } \\
\text { federativa }\end{array}$ & Comunidades & $\%$ & $\begin{array}{r}\text { Superficie } \\
\text { (has) }\end{array}$ & $\begin{array}{r}\% \\
\text { Superficie } \\
\text { promedio } \\
\text { (has) }\end{array}$ \\
\hline Baja California & 3 & 2.06 & 221619 & 9.80 & 73873.00 \\
Chihuahua & 75 & 51.17 & 590800 & 26.12 & 7877.33 \\
Coahuila & 2 & 1.37 & 11504 & 0.51 & 5752.00 \\
Nuevo León & 14 & 9.65 & 123996 & 5.50 & 8856.85 \\
Sonora & 47 & 32.41 & 1133639 & 50.13 & 24119.97 \\
Tamaulipas & 4 & 2.75 & 179765 & 7.94 & 44941.25 \\
\hline Total & 145 & 100.0 & 2261323 & 100.0 & 15595.33 \\
\hline
\end{tabular}

Fuente: Elaboración propia con datos del Registro Agrario $\mathrm{Nacional,}$ Subsecretaría de Ordenamiento de la Propiedad Rural, septiembre de 2000. http:/ / www.sra.gob.mx/ monag/ bcweb/ s2Bajacalifornia.htm http:/ / www.sra.gob.mx/ monag/ coahweb/ s2Coahuilal.htm http:/ / www.sra.gob.mx/ monag/ chiweb/ s2Chihuahua.htm http:/ / www.sra.gob.mx/ monag/ nlweb/ s2N uevo\%20Leon1.htm http:/ / www.sra.gob.mx/ monag/ tamweb/ s2\%20Tamaulipas.htm http:/ / www.sra.gob.mx/ monag/ sonweb/ s2Sonora2.htm 
embargo, en cuanto a la calidad delos recursos natural es sobresaIen las comunidades de Baja California, Sonora y Chihuahua.

En cuanto a las colonias agrícolas y ganaderas, en la región existen 375 colonias con una superficie de 2015763 hectáreas y una superficie promedio por colonia de 5375hectáreas. Demanera segregada en cada uno delos estados dela región, se puedeobservar en el cuadro 9 que los estados de Sonora, Chihuahua y Baja California, en forma conjunta, absorben $86 \%$ delas colonias agrícolas y ganaderas; en cambio, en cuanto al giro de la actividad, se puede decir queen las col onias de Baja Cal ifornia y Sonora predomina la agricultura y en el resto de los estados la ganadería.

En estecontexto, seinfiere quelas propiedades social y privada siguen integrando la estructura productiva de la frontera norte, a pesar de los cambios en la política agropecuaria y agraria queindicaban una tendencia a la privatización de la propiedad social. El cuadro 10 muestra que las ventas detierras ejidales en la frontera nortees cercana a $8 \%$, y esta superficievendida se ubica principalmenteen aquel los ejidos conurbados a las ciudades, por lo que no

CUADRO 9. Número de colonias agrícolas y ganaderas en la frontera norte y participación por entidad federativa de la superficie.

\begin{tabular}{|lrrrrr|}
\hline $\begin{array}{l}\text { Entidad } \\
\text { federativa }\end{array}$ & Colonias & $\%$ & $\begin{array}{r}\text { Superficie } \\
\text { (has) }\end{array}$ & $\begin{array}{r}\text { \% } \\
\text { Superficie } \\
\text { promedio } \\
\text { (has) }\end{array}$ \\
\hline Baja California & 103 & 27.46 & 236686 & 11.74 & 2297.92 \\
Chihuahua & 47 & 12.53 & 572872 & 28.42 & 12188.76 \\
Coahuila & 20 & 5.33 & 434484 & 21.55 & 21724.20 \\
Nuevo León & 7 & 1.86 & 117915 & 5.87 & 16845.00 \\
Sonora & 173 & 46.13 & 346501 & 17.18 & 2002.89 \\
Tamaulipas & 25 & 6.66 & 307305 & 15.24 & 12292.20 \\
\hline Total & 375 & 100.0 & 2015763 & 100.0 & 5375.36 \\
\hline
\end{tabular}

Fuente: Elaboración propia con datos del Registro Agrario NacionalSubsecretaría de Ordenamiento de la Propiedad Rural. http:/ / www.sra.gob.mx/ monag/ bcweb/ s2Bajacalifornia.htm http:/ / www.sra.gob.mx/ monag/ coahweb/ s2Coahuilal.htm http:/ / www.sra.gob.mx/ monag/ chiweb/ s2Chihuahua.htm http:/ / www.sra.gob.mx/ monag/ nlweb/ s2Nuevo\%20Leon1.htm http:/ / www.sra.gob.mx/ monag/ tamweb/ s2\%20Tamaulipas.htm http:/ / www.sra.gob.mx/ monag/ sonweb/ s2Sonora2.htm 
CuADRo 10. Formas de acceso a la tierra en la frontera norte.

\begin{tabular}{|lrrr|}
\hline $\begin{array}{c}\text { Entidad } \\
\text { federativa }\end{array}$ & $\begin{array}{r}\text { Herencia o cesión } \\
\text { a familiares (\%) }\end{array}$ & $\begin{array}{c}\text { Compra } \\
(\mathbf{\%})\end{array}$ & $\begin{array}{c}\text { O tros* } \\
\text { (\%) }\end{array}$ \\
\hline Baja California & 73.40 & 6.70 & 19.90 \\
Chihuahua & 43.60 & 8.50 & 47.90 \\
Coahuila & 55.20 & 3.80 & 41.00 \\
Nuevo León & 27.30 & 9.60 & 63.10 \\
Sonora & 47.50 & 3.80 & 48.70 \\
Tamaulipas & 34.50 & 14.7 & 50.80 \\
\hline Total promedio & 46.91 & 7.85 & 45.23 \\
\hline
\end{tabular}

Fuente: Procuraduría Agraria.

*Entre ellos la acción agraria, adjudicación y acuerdo por asamblea.

http:/ / www.sra.gob.mx/ monag/ bcweb/ s3abcn.htm http:/ / www.sra.gob.mx/ monag/ coahweb/ s3acoa.htm http:/ / www.sra.gob.mx/ monag/ chiweb/ s3achi.htm http:/ / www.sra.gob.mx/ monag/ nlweb/ s3anl.htm http:/ / www.sra.gob.mx/ monag/ tamweb/ s3atam.htm http:/ / www.sra.gob.mx/ monag/ sonweb/ s3ason.htm

es significativo en términos de las actividades rurales (datos de la Procuraduría Agraria, 2000).

\section{CONCLUSIONES Y RECOMENDACIONES}

El capital privado nacional y extranjero no llegó al campo en los términos que el Estado esperaba, por lo que los productores del sector social y privado no lograron insertarseen los proyectos especializados que se canalizan a la exportación, generando una descapitalización en los pequeños, medianos y grandes productoresque se dedicaban a la producción para el mercado interno. Los proyectos deasociación entreel capital privado nacional y extranjero con los ejidos y comunidades agrarias que promovió el Estado en aquellas zonas quecuentan con tierras de buena calidad, agua e infraestructura, sólo duraron entre dos y tres años, ya que funcionaron mientras el Estado otorgaba apoyos económicos, como es el caso del Proyecto Vaquerías en el estado deN uevo León, en el que 
se asociaron la empresa Galletera M exicana S. A . y los ejidos productores detrigo con la finalidad de producir materia prima para la empresa Gamesa.

En las zonas agrícolas donde ya estaba presente el capital extranjero, con la reforma al artículo 27 , solamente amplió sus proyectos agroindustriales, como es el caso del sur deSonora y el Valle de Mexicali, zonas en las cuales en lugar de presentarse proyectos de asociación se presentó la renta de la tierra a mayor escala, haciendo a un lado los proyectos de asociación con los ejidos y las comunidades agrarias.

A nte esta situación, el Estado se vio obligado a formular y aplicar políticas públicas compensatorias tales como el Programa de A poyo al Campo (Procampo) y el Programa de Alianza para el Campo, entreotras, mismasqueno han logrado resolver los problemas de descapitalización de los productores del sector social y privado.

En la frontera nortela propiedad social siguesiendo importante y seguirá existiendo por mucho tiempo a pesar delos cambios en la política agropecuaria y sobre todo con los cambios al artículo 27 constitucional deenero de 1992 y a la promulgación dela Ley Agraria en febrero del mismo año, que abre al mercado la propiedad social rural. El mercado de tierras dela propiedad social rural no ha sido significativo, ya que la venta de tierras en la frontera norte es cercana al $8 \%$ de la superficie total, y esta superficie se ubica principalmente en aquellos ejidos y comunidades agrarias conurbados a las ciudades, por lo que no es significativo en términos de las actividades agropecuarias. La propiedad social seguirá existiendo en diversas modalidades: en una primera modalidad se encuentran los ejidos y comunidades agrarias que aún trabajan la tierra directamentey dependen deella como una fuentedeingresos importante; en una segunda modal idad seencuentran los ejidosy las comunidades agrarias que por condiciones de ubicación geográfica y sistemas de producción para la exportación prefieren rentar sus tierras al capital privado nacional o extranjero, dados los escasos apoyos que otorga el Estado; sin embargo, la propiedad 
sigue perteneciendo al régimen depropiedad social, es decir, están dentro del Registro A grario Nacional. Esta propiedad social rentada al capital privado seguirá siendo propiedad social, mientras no cambie su registro, es decir, al Registro Público de la Propiedad.

La organización delos productores, tanto del sector social como del privado, está en crisis y la mayoría de los productores trabajan con sus propios recursos y demanera individual. Esto ha dificultado la integración de proyectos productivos, y los programas de organización y capacitación que han promovido las instituciones del sector agrario, como la Secretaría de la Reforma A graria y la Procuraduría A graria, no han tenido éxito debido a la falta de recursos financieros y personal capacitado para la formulación y evaluación deproyectos rentables.

A nte esta situación de crisis en el campo dela frontera norte, el sector rural no es capaz de proporcionar empleos a toda la población asentada en el medio rural, por lo que los productores del sector social y sus familias se ven obligados a incursionar en el mercado detrabajo en otros sectores fuera del campo: la industria maquiladora, la construcción en las ciudades cercanas a las comunidades y la migración demanera temporal hacia Estados Unidos.

Es necesaria la intervención del Estado a través de políticas y programas, inversión pública en infraestructura que contribuya a resolver estos problemas y mejorar el desarrollo productivo del sector agrícola y rural, a la vez quelas familias rurales puedan aumentar sus ingresos y con ello mejorar sus niveles devida.

\section{BiBLIOGRAFÍA}

A ppendini, Kirsten. 1995. “La transformación dela vida económica en el campo mexicano", en Jean Francois Pru'homme (coord.) El impacto social de las políticas de ajuste en el campo mexicano, Ed. Plaza y Valdés, México, D.F.

Cartón deGrammont, Hubert. 1996, “Política agraria y estructura territorial”, Revista de Estudios A grarios, núm. 2, enero-marzo, Procuraduría A graria, México, D.F. 
Encinas, Alejandro, et al. 1995. "M ovimiento campesino y reforma neoliberal", en Alejandro Encinas (coord.), El campo mexicano en el umbral del sigloXXI, Espasa-Calpe, México.

Fritscher, Magda. 1993. "La reforma agrícola del salinismo", en Pedro Castro (coord.), L as políticas sal inistas: balancea mitad de sexenio (1988-1991), Universidad Autónoma MetropolitanaIztapalapa, México.

Gómez Olivier, Luis. 1996. “El papel dela agricultura en el desarroIlo de México", Revista de Estudios A grarios, núm. 3, abril-junio, Procuraduría Agraria, México.

Gordillo, Gustavo. 1991. “Raíz y razón delas reformasal A rtículo 27 Constitucional", La Jornada, 8denoviembre, México.

Instituto Nacional de Estadística, Geografía e Informática. 2000. X II Censo General dePoblación y V ivienda. Resultados preliminares, Aguascalientes, M éxico.

Salinas de Gortari, Carlos. 1991. "Iniciativa de Reforma al Artículo 27 Constitucional", Comercio Exterior (Banco Nacional deComercio Exterior), vol. 41, núm. 11, noviembre, México.

Secretaría de la Reforma A graria. 2002. Programa de D esarrollo Regional Frontera $\mathrm{N}$ orte 2001-2006.

Téllez, Luis. 1994. La modernización del sector agropecuario y forestal, Fondo de Cultura Económica, México.

Warman, Arturo. 1996. "La reformaal Artículo 27 Constitucional”,

R evista Estudios A grarios, núm. 2, enero-marzo, Procuraduría Agraria, México.

Zermeño, Felipe. 1992. “La agricultura mexicana anteel Tratado de Libre Comercio", en Alejandro Encinas, Juan de la Fuentey Horacio Mackinlay (coords.), La disputa por los mercados, TLC y sector.

Entrevistas a líderes campesinos

N oéTapia, presidentedel ComitéRegional Campesino del Municipio deMatamoros, Tamaulipas (marzo de 2002). 
José Luis Razo, presidente del Comité Regional Campesino del Municipio de Caborca, Sonora (abril de 2002).

Gildardo Duarte, presidente del Comité Regional Campesino del Municipio deA gua Prieta, Sonora (abril de 2002).

Leonel Fal cón Garza, presidente del Comité Regional Campesino del Municipio de Reynosa, Tamaulipas (marzo de 2002).

LuisA raujo, presidentedel ComitéRegional Campesino del Municipio de N ogales, Sonora (abril de 2002).

Pedro Mireles López, presidentedel ComitéRegional Campesino del Municipio deLampazos, Nuevo León (marzo de 2002).

GuadalupeMorín, presidentedel ComitéRegional Campesino del Municipio deCamargo, Tamaulipas (abril de2002).

Gonzalo González, presidentedel ComitéRegional Campesino del Municipio de A náhuac, Nuevo León (abril de 2002).

Epifanio Martínez, presidentedel ComitéRegional Campesino del Municipio deJiménez, Coahuila (marzo de 2002). 